\title{
Reformulating Non-Monotonic Theories For Inference and Updating
}

\author{
Benjamin N. Grosof \\ IBM T. J. Watson Research Center \\ P.O. Box 704, Yorktown Heights, NY 10598 \\ (914) 784-7100 ; Internet: grosof@watson.ibm.com
}

\begin{abstract}
We aim to help build programs that do large-scale, expressive non-monotonic reasoning (NMR): especially, "learning agents" that store, and revise, a body of conclusions while continually acquiring new, possibly defeasible, premise beliefs. Currently available procedures for forward inference and belief revision are exhaustive, and thus impractical: they compute the entire non-monotonic theory, then re-compute from scratch upon updating with new axioms. These methods are thus badly intractable. In most theories of interest, even backward reasoning is combinatoric (at least NP-hard). Here, we give theoretical results for prioritized circumscription that show how to reformulate default theories so as to make forward inference be selective, as well as concurrent; and to restrict belief revision to a part of the theory. We elaborate a detailed divide-and-conquer strategy. We develop concepts of structure in NM theories, by showing how to reformulate them in a particular fashion: to be conjunctively decomposed into a collection of smaller "part" theories. We identify two well-behaved special cases that are easily recognized in terms of syntactic properties: disjoint appearances of predicates, and disjoint appearances of individuals (terms). As part of this, we also definitionally reformulate the global axioms, one by one, in addition to applying decomposition. We identify a broad class of prioritized default theories, generalizing default inheritance, for which our results especially bear fruit. For this asocially monadic clase, decomposition permits reasoning to be localized to individuals (ground terms), and reduced to propositional. Our reformulation methods are implementable in polynomial time, and apply to several other NM formalisms beyond circumscription.
\end{abstract}

\section{Introduction}

Large-Scale, Expressively Rich, Learning
Agents: We aim in this work ${ }^{1}$ to help build agents that do large scale, expressive non-monotonic reasoning (NMR). We are interested especially in what we call learning agents: automatic programs that store, and revise, a body of conclusions while continually acquiring new, possibly defeasible, premise beliefs.

In many applications, information about which defaults take precedence over others (have greater prioritization) is important and available. ${ }^{2}$ Many applications need the ability to express fairly arbitrary first-order forms of default beliefs (e.g., induction, law, natural language, communication), as well as fairly arbitrary (finite) partial orders of precedence (e.g., specificity, reliability, and authority are not "layered" (a.k.a. "stratified"))). [Grosof, 1991] defines and discusses the importance of non-layered priority. Non-layered priority is needed, for example, to adequately represent default inheritance.

In these applications, we regard as desirable for many reasons, especially validation (both intuitive and rigorous), that a NM formalism be "expressively rich" not only in the above senses, but also that it be equipped with a relatively strong model-theoretic semantics (e.t., cf. Default Logic [Reiter, 1980], circumscription [McCarthy, 1986] [Lifschitz, 1984], and Autoepistemic Logic [Moore, 1985]). In this connection, we also are interested in skeptical or cautious, rather than credulous or brave, entailment.

Current Incapabilities: Currently, expressively rich $\mathrm{NMR}^{3}$ has found virtually no application on a large scale (more than order of ten defaults), except for the rather special cases of Prolog-style logic programs and simple inheritance cf. AI frame-based systems.

Part of the problem is that there do not yet ex-

\footnotetext{
${ }^{1}$ part of forthcoming PhD discertation [Grosof, 1992b]

${ }^{2}$ Note, however, that most of the discussion and results, e.g., about disjoint describability and definitional reformulation and asocially monadic theories, in this paper also apply to the basic case where there are only two "priority levels": for-sure and defeanible.

${ }^{3}$ In [Grosof, 1992b], we make this more precise; here, let us just consider circumscription, Default Logic, and Autoepistemic Logic.
} 
ist practical inference mechanisms to support storing and revising a limited body of conclusions as a working theory. Currently, for expressively rich NMR 4 , the only procedures for forward ${ }^{5}$ inference are exhaustive: they compute the entire non-monotonic theory (or, even worse, all credulous extensions). Also, currently, there are no procedures for performing belief revision on a body of conclusions, upon receiving new, asserted axioms (an update), beyond the exhaustive method of re-computing everything from scratch. (By "axiom", we mean a premise belief.) ${ }^{6}$ By belief revision, we mean modifying the stored conclusions to retract those that are no longer entailed by the newly augmented axiom set. ${ }^{7}$ By updating, we mean belief revision plus possibly the inference and storing of some additional conclusions.

Strategy and Summary: In this work, we attack these problems at the level of logical understanding (rather than, say, domain-dependent control of reasoning). Our analytic perspective is that a prime underlying difficulty in the tasks of inference and updating, as well as in specification, is the logical globality of NMR: in general, conclusions depend on the whole of the axiom set. The exhaustiveness of current methods is, in effect, a manifestation of their caution in dealing with (conflicting) interaction.

We define the concept of a prioritized database, using circumscription, as the logical representation of a learning agent that performs sound, but incomplete, expressively rich NMR. By database, we mean a subset of a (NM) theory. Prioritized circumscription meets our prime expressive concerns, offers mathematical convenience, and has inference procedures currently available.

We elaborate a detailed "divide and conquer" strategy. We develop concepts of, and results about, structure in prioritized circumscriptive theories, by showing how to reformulate them in a particular fashion: to be conjunctively "decomposed" hierarchically into a collection of smaller "part" theories, i.e., sub-theories which we call slices. We show that it is possible, and useful, to slice within slices. In this way, we map groups of axioms to groups of conclusions. We use the decompositions to analyze the interaction between defaults / parts in a NM theory. Much technical diffculty and trickiness arises from the expressive need to consider non-layered prioritization.

We give theorems that localize entailment and thus show how to make forward inference be selective, as

\footnotetext{
including even the propositional special case and the special case of stratified logic programs with negation [Lifschitz, 1987] [Przymusinski, 1988]

sbottom-up. By "backward", we mean totally goaldirected $c f$. query-answering.

'NM formalisms, e.g., JTMS's [Doyle, 1979], having such procedures lack our desired expressive properties.

${ }^{7}$ For simplicity, we assume that these are the only ones removed from storage.
}

well as concurrent. Exhaustive inference on a slice generates only a part of the global theory. Inferences within each slice (sub-theory) can be performed in parallel with inference within every other slice. All nonmonotonic inference can be localized to the slices; only monotonic inference is required between the slices. We give theorems that localize retraction and thus show how to make belief revision be partial in the sense that, for a given update, the arena of potential retraction is known to be restricted to a particular part of the previous database.

Our results enable the exploitation of other results on inference and belief revision that are limited to expreseive special cases, say to do exhaustive forward inference in polynomial time (e.g., the "sympatheticsolitary" case in [Grosof, 1992b] that generalizes predicate completion [Clark, 1978] and the Closed World Assumption). These special case results can be applied to one, or several, slices, even when they do not apply to the global theory.

Our results are about well-behaved special cases that are easily recognized in terms of syntactic properties. The first "cleanly slice-able" property is disjointness of mentioned predicates. We show that if the for-sure and default axioms can be partitioned into groups which are disjoint in terms of the predicate symbols they mention, then non-monotonic inference based on each partition can proceed without considering the axioms in the other partitions: those other axioms are irrelevant in an important sense, as far as that partition is concerned. We show this implies that updating with new for-sure and default axioms that span only some of the previous partitions does not require retracting previous conclusions based purely on the remaining partitions: they are safe.

Most large practical applications, however, do not display such perfect partitionability of mentioned predicates. The real power from our result about disjointness of predicates comes when it is combined with another kind of reformulation: of the axioms in a given global axiom set, not just of the global axiom set into decomposed constituent axiom sets. We define a concept of disjoint describability: syntactic partitionability after definitional reformulation of the axioms. As part of this, we give a logical definition of a particular kind of definitional (i.e., equivalence-preserving) reformulation with respect to a background theory, modifying the standard logical idea of a conservative extension. We also discuss, and use, another kind of reformulation: to break up open defaults (i.e., schema-type, as opposed to closed, i.e., propositional) into cases. An important difference from definitionally reformulating monotonic theories is that two default axioms $D 1$ and $D 2$ cannot, in general, be equivalently replaced by the default axiom corresponding to the conjunction $D 1 \wedge D 2$ the way that two for-sure axioms can always be equivalently replaced by their conjunction $B 1 \wedge B 2$. This is why we need to consider reformulation of the 
axioms one-by-one.

Using these definitional and default-cases reformulations, we arrive at our second cleanly slice-able, yet syntactically recognizable, property: disjointness of mentioned individuals. We show that a fairly broad class ("asocially monadic") of prioritized default circumscriptions is cleanly slice-able into one slice theory per named individual (ground term in the language) plus a remainder-case slice. Each of these individual-wise slices is propositional, and is, essentially, much simpler than the global, in several ways: number of axiom instances (especially, potential primitive default conclusions), availability of inference methods, and availability of known computational complexity results. (Unfortunately, we do not have space to discuss NM inference methods and complexity results in detail. But see the final section.) The asocially monadic class includes, as a special case, default inheritance networks of the kind studied by [Touretzky, 1986] and used in many AI frame-based systems. The asocially monadic class is more general, however: it permits more than one antecedent in default rules, free use of negation, and freer use of disjunction.

While definitional reformulation is hard in general, we have a polynomial-time algorithm (omitted in this draft to save space and preserve focus) to peform the recognition and exploitation of this asocialmonadic reformulation and decomposition. More precisely, the algorithm is $O\left(n^{3}\right)$, where $n$ is the size of the (global) axiom set (which is, moreover, typically much smaller than the whole theory, of course).

We show that our conjunctive decomposition results imply safeties in belief revision. We illustrate the problems of scale in learning agents with an extended example of a prioritized database and show that our safety theorems capture much of the preponderant stability (i.e., most beliefs are preserved after each update) that this database displays through its sequence of updates. We show, using the example, that decompositions on these two bases combine synergistically, as well as hierarchically: it is useful to slice within slices.

Finally, we observe that our formal reformulation methods are implementable at reasonable cost, and apply to several other NM formalisms. We have polynomial-time algorithms (again, omitted here due to space and focus) for disjoint predicates, as well as for asocially monadic, also in $O\left(n^{3}\right)$, where $n$ is the size of the (global) axiom set.

\section{A Motivating Example}

Next, we give an extended example of a learning agent, in the domain of common-sense default reasoning, that illustrates issues of selective forward inference and partial belief revision on a large scale. We present it first at an intuitive level, and formalize it later.

We adopt the following notation. A $\bullet>$ prefix indicates that the sentence that follows is a base axiom, i.e., has for-sure (non-defeasible) belief status. $A$ :> pre- fix indicates that the formula that follows is a default axiom (roughly, a normal default without pre-requisite in Default Logic). Its label, e.g. (d1), serves as a tag for defining prioritization-type precedence between defaults via $\mathcal{P} \mathcal{R E F E R}$ (prioritization) axioms. These define a strict partial order of precedence, via transitive closure. $\mathcal{P} \mathcal{R E F} \mathcal{E} \mathcal{R}(d 1, d 2)$, for example, means that the default axiom with label $(d 1)$ has strictly greater precedence (priority) than the default axiom with label (d2).

We make the Uniqueness of Names Assumption (consider it included as a for-sure axiom). As a shorthand for conjunctions of for-sure assertions of positive or negative literals, we list the satisfying objects, or, more generally, tuples. Often, in this context, we use "..." to indicate that there are additional satisfying tuples not shown explicitly; for simplicity's sake, we assume these objects are distinct from all other explicitly-shown objects.

In this example, the agent starts with no beliefs, then accumulates axioms by receiving updates. After each update, the agent draws a bunch of conclusions (say, ground first-order sentences), both monotonically and non-monotonically, and retracts some of its previous conclusions. Each $u_{i}$ indicates an update, consisting of one or more axioms. Axioms are numbered. In addition, we show explicitly with $\approx$ and $\forall \neq$ a few of the more interesting NM conclusions and retractions, respectively, about which discussion will revolve. Note that, by "conclusion", we always mean in the skeptical sense.

The first update consists of a default axiom, that bats have two legs, together with some for-sure axioms. Non-monotonic (default) conclusions include that known bats are two-legged. The second update consists of another, default axiom, that mammals have four : $-\mathrm{gs}$, together with the precedence axiom, that this new fault has lower priority than the previous, more specinc one. The third update consists of two default axioms about emergency disaster situations, plus some associated for-sure information. Intuitively, since the axioms in this new update are about a totally different topic than the previous axioms, they should not result in having to retract any of the previous conclusions. Moreover, intuitively, the agent should be able to draw the conclusions from these new axioms without even having to consider the previous ones in detail.

The fourth update consists of some for-sure information about two named individuals, Joe and Spot, that violates some previous default conclusions. Intuitively, since there is no information that "connects" any other named individuals to $J o e$ and $S$ pot, these new axioms should not result in having to retract any of the previous conclusions that are not about those named individuals: e.g., that are about some other named individuals. For example, the previous default conclusion 2 legs(Betsy) should not have to be retracted.

Later, we will show how to capture these intuitions 
Example's Axioms and Sample Conclusions

$\mathcal{U}_{1}$ : Mammals Taxonomy plus: Bats are Two-Legged

[1] $\bullet>\forall x . \operatorname{bat}(x) \supset$ mammal $(x)$

[2] $\quad>\forall x \cdot \operatorname{dog}(x) \supset \operatorname{mammal}(x)$

[3] •> +bat : Betsy, Joe, June, Jackie, ...

[4] •> +dog: Fido, Spot, Siccem, Jumper,...

[5] $\bullet \forall x . \neg(2 \operatorname{legs}(x) \wedge 4 \operatorname{legs}(x))$

[6] (d1) :> bat $(x) \supset 2$ legs $(x)$

$\&_{i} \mathcal{U}_{i} \quad \approx 2$ legs $($ Betsy $) \wedge 2$ legs $($ Joe $) \wedge \ldots$

$\mathcal{U}_{2}$ : Lower-Priority Default about Legged-ness

[7] (d2) :> mammal $(x) \supset 4 \operatorname{legs}(x)$

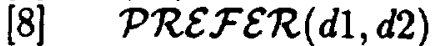

$\&_{i} \mathcal{U}_{i} \quad \approx$ Plegs $($ Fido $) \wedge 4$ legs $($ Spot $) \wedge \ldots$

$\mathcal{U}_{3}$ : Emergencies (cf. [Grosof, 1991])

[9] (d3) :> fire (place, day $) \wedge$ person $(x) \supset$ leave $(x$, place, day $)$

[10] (d4) :> earthquake(place, day) $\wedge$ person $(x) \supset$ leave $(x$, place, day $)$

[11] $\bullet>$ tperson: Sue, Andy, Ed, Peg, Maggie, Eileen, Chang,...

[12] $\bullet\rangle$ +fire: $\langle$ Baltimore, $2 / 4 / 03\rangle,\langle$ Watts, $8 / 2 / 67\rangle, \ldots$

[13] $\bullet>$ tearthquake: $\langle S F, 4 / 8 / 06\rangle,\langle$ MexicoCity, $5 / 3 / 87\rangle, \ldots$

[14] $\bullet \forall x$, place, day. leave (x, place, day) $\supset \neg$ attend_work $(x$, place, day $)$

$\& \mathcal{U}_{i} \quad \approx$ leave $($ Sue $, S F, 4 / 8 / 06) \wedge$ leave $($ Andy, Watts, $8 / 2 / 67) \wedge \ldots$

$\mathcal{U}_{4}$ : Legged-ness: Selective Defeat For Individuals

[15] •> $\neg 2$ legs $($ Joe $) \wedge \neg 4$ legs (Joe) $\wedge \neg 2$ legs $($ Spot $) \wedge \neg 4$ legs $($ Spot $)$

$\&_{i} \mathcal{U}_{i} \quad \not \forall 2$ legs(Joe) ; $\forall$ 4legs(Spot)

$\mathcal{U}_{5}$ : Work Attendance (cf. [Grosof, 1991])

[16] (d6) :> weekday $(d) \wedge$ reg_employ $($ person,place $) \supset$ attend_work (person, place, $d)$

[17] (d7) :> flu(person,day) つ حattend_work(person, place,day)

[18] $\mathcal{P R E F \mathcal { R }}(d 7, d 6)$

[19] $\operatorname{PREFER}(d 3, d 7) \& \mathcal{P R E F E R}(d 4, d 7) \& \mathcal{P R E F E R}(d 5, d 7)$

$\mathcal{U}_{6}$ : Ed is Ill; Conflict Resolved by Prioritization (cf. [Grosof, 1991])

[20] •> +weekday: Today, $11 / 12 / 91, \ldots$

[21] $\bullet>$ +reg_employ: $\langle E d, B l d g A\rangle, \ldots$

[22] $\bullet>+f l u:\langle E d$, Today $\rangle, \ldots$

$\&_{i} \mathcal{U}_{i} \quad \approx$ ᄀattend_work $(E d$, Today)

$\mathcal{U}_{7}:$ Miscellany: Meetings and Attendance (cf. [Grosof, 1991])

[23] -> +Tuesday: Today, 11/12/91,...

[24] •> +in_group $(p, 4321): E d$, Peg, Maggie,...

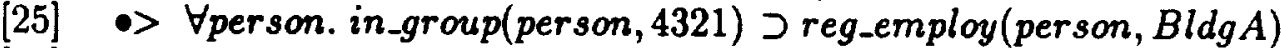

[26] •> ᄀvacation(Boss $(4321), d):$ Today,...

[27] •> $\forall p, d$. group_meeting $(p, d) \wedge$ in_group $(p, 4321) \supset$ attend_work $(p, B l d g A, d)$

$\mathcal{U}_{8}$ : Group Meetings; Non-Layered Conflict (cf. [Grosof, 1991])

[28] $(d 9):>$ in_group $(p, 4321) \wedge$ Tuesday $(d) \supset$ group_meeting $(p, B l d g A, d)$

[29] $(d 10):>$ in_group $(p, 4321) \wedge$ vacation $(B o s s(4321), d) \supset$ ᄀgroup_meeting $(p, B \operatorname{Blg} A, d)$

[30] $\mathcal{P} \mathcal{R E} \mathcal{F E R}(d 10, d 9)$

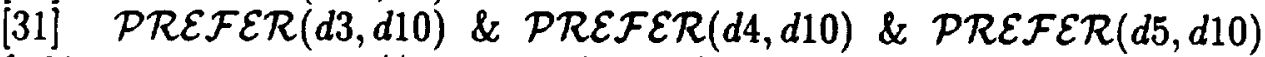

$\&_{i} \mathcal{U}_{i} \quad \not$ attend_work(Ed,Today) ; $\not E$ attend_work(Ed,Today) 
as formal guarantees.

\section{Formal Definitions: Prioritized Circumscription}

We define our notation for axioms from section 2 as a meta-language (the Circumscriptive Language of Defaults, or CLD for short) that, at any point in the update sequence, specifies a prioritized "default" circumscription of the form:

$$
\begin{aligned}
& P D C(B ; D ; R ; \text { fix } W ; Z) \stackrel{\text { def }}{\equiv} \\
& B[Z] \wedge \neg \exists Z^{\prime} . B\left[Z^{\prime}\right] \wedge Z \prec_{(D ; R)} Z^{\prime} \wedge W=W^{\prime}
\end{aligned}
$$

Here, $B$ is the conjunction of the sentence parts of all of the for-sure axioms. $D$ is the tuple of the default axioms' formula parts. $R$ is a strict partial order of precedence (priority). It is the transitive closure of the precedence relation specified by the pairwise comparisons in the $\mathcal{P} \mathcal{R E F} \mathcal{F} \mathcal{R}$ axioms. Its domain, accordingly, is the set of default axiom labels. $Z$ is the tuple of all mentioned predicate symbols; e.g., in the example, (bat, dog, mammal, 2legs, 4legs, fire,...). $W \subset Z$ is the tuple of predicates that are fixed. Fixing is a standard notion in the circumscription and non-monotonic reasoning literature. Fixing is part of the specification of non-monotonic reasoning. Intuitively, fixing some symbols implies that any formula that mentions only those symbols is immune to the circumscription operation in the sense that it can be concluded nonmonotonically, i.e., from the circumscription, only if it can be concluded "monotonically", i.e., from the forsure axioms $B$ alone. For simplicity, we also fix (do not vary and second-order quantify over) all function symbols. This assumption can easily be relaxed. This assumption is typical in the circumscription literature. Uniqueness of Names, plus Domain Closure, implies that functions are effectively fixed, for example. For the sake of simplicity, in this paper, we for the most part do not consider fixing of predicates, only of functions: $W$ is empty. We omit further details about fixing to save space and to preserve focus; see [Grosof, 1992b] for more.

Prioritized default circumscription is a slight generalization of prioritized predicate circumscription $\mathrm{cf}$. [Grosof, 1991]. We employ it and CLD to clarify the definitions of axiom sets and of updating, and the intuitive relationship to other formalisms for default reasoning. [Grosof, 1992b] shows as a theorem the equivalence of any prioritized default circumscription to a corresponding, abnormality-style, prioritized predicate circumscription, generalizing a previous result that appeared in [Lifschitz, 1984]. Note that our definition can express minimizing predicates as a special case: e.g., :> $a b_{i}(x)$, where $a b_{i}$ is an abnormality predicate.

We let $N$ stand for the index tuple of $D$ : it is just (isomorphic to) the tuple of the labels of the default axioms. I.e., in the example, after the second update, the elements of $D[Z]$ are:

$$
\lambda x . \operatorname{bat}(x) \supset \operatorname{legs} 2(x) \text {, }
$$

$\lambda x$. mammal $(x) \supset 4 \operatorname{legs}(x)$

and $N=\langle d 1, d 2\rangle . R(j, i)$ means that the default with label $j$ has strictly higher priority than the default with label $i . \quad \prec(D ; R)$ is defined as the strict version $(\preceq(D ; R) \wedge \neg \succeq(D ; R))$ of the prioritized "formula" preorder $\preceq(D ; R)$ :

$$
\begin{aligned}
Z \preceq(D ; R) & Z^{\prime} \stackrel{\text { def }}{\equiv} \\
\forall i \in N .[\forall j & \in N . R(j, i) \supset \\
& \left.\left(\forall x . D j[Z, x] \equiv D j\left[Z^{\prime}, x\right]\right)\right] \\
& \supset\left(\forall x . D i[Z, x] \supset D i\left[Z^{\prime}, x\right]\right)
\end{aligned}
$$

Here $D j$ and $D i$ refer to the $j^{t h}$ and $i^{t h}$ members, respectively, of the tuple $D^{8}$ We define the corresponding circumscriptive prioritized default theory as the set of all conclusions entailed (model-theoretically, in second-order logic) by the prioritized default circumscription. 910 We define a prioritized database PDB) to be a pair, consisting of $\{$ CLD axiom set $\mathcal{A}$ in the example, the current coli- ion of the updates idi's)); and an associated prioritied database theory $D B$, which is some subset of the cumscriptive theory $\mathcal{C}(\mathcal{A})$ specifie $\perp$ by $\mathcal{A}$. Here, $\mathcal{C}$ is the non-monotonic theory operator for the CLD formalism.

\section{Decomposition: Concepts}

As part of our strategy, we need to develop a strong idea of a part of a non-monotonic theory. This is important for several reasons: 1) to define safe versus unsafe zones for belief revision; 2) to define relevant versus irrelevant context for inference (and for specification); and 3) to define the structure and organization of an overall ("global") prioritized database. In classical logic, we take for granted such an idea of a part of theory. However, the dependence of entailment on, in general, the entire global axiom set means that we have to "work for it" in NM logical systems.

Our general concept of decomposition is applicable to many NM logical systems. A global theory $\mathcal{T}$ can be obtained either directly by applying the NM theory operator $\mathcal{C}$ to the global axiom set $\mathcal{A}$, or indirectly (but equivalently) via decomposition. In decomposition, the global axiom set $\mathcal{A}$ is decomposed into an associated set of "constituent" axiom sets (the $S \mathcal{A}_{i}$ 's). The global theory $\mathcal{T}$ is then equivalent to the combi. nation of the corresponding sub-theories (the $S T_{i}$ 's), where each sub-theory is the result of applying $\mathcal{C}$ to a constituent axiom set: $S \mathcal{T}_{i} \stackrel{\text { def }}{=} \mathcal{C}\left(\mathcal{S} \mathcal{A}_{i}\right)$.

\footnotetext{
'For notational simplicity, we ignore the potentially different arities of the various open formulas $D i$.

${ }^{\circ}$ See [Grosof, 1991] and [Grosof, 1992b] for more discussion of how prioritized circumscriptions are defined. Note that the prioritization p.o. $R$ is not necessarily layered (stratified) (indeed, in our example, it is not) as it was in [Lifachitz, 1985].

${ }^{10}$ In section 5 , we generalize the definition above to include the explicit "fixing" of a set of formulas, e.g., a subset of the predicates. [Grosof, 1992b] gives details.
} 


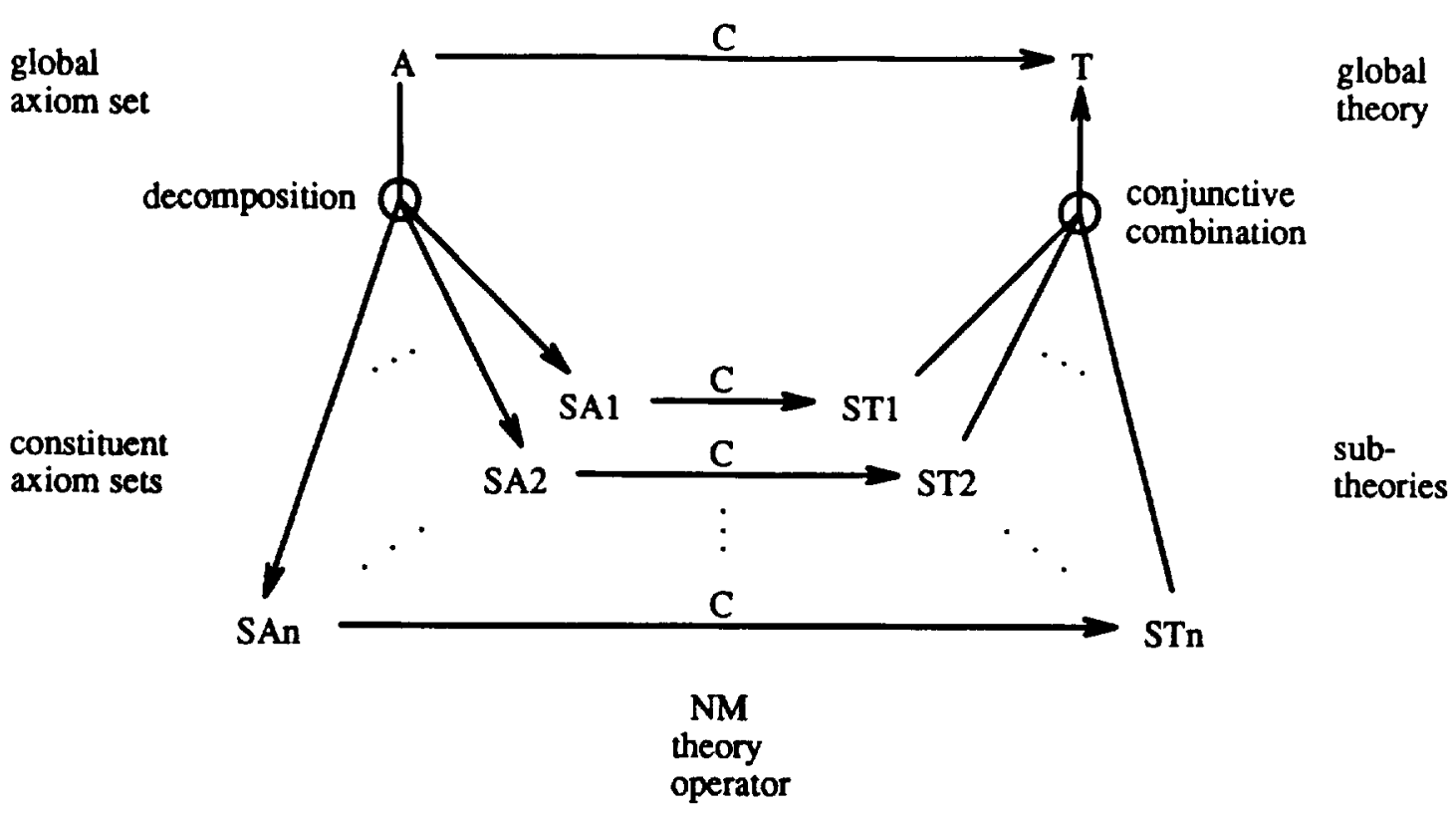

Figure 1: Conjunctive Decomposition: a conceptual flow diagram. A global theory $\mathcal{T}$ can be obtained either directly by applying the NM theory operator $\mathcal{C}$ to the global axiom set $\mathcal{A}$, or indirectly (but equivalently) via decomposition. In decomposition, the global axiom set $\mathcal{A}$ is decomposed into an associated set of constituent axiom sets (the $\mathcal{S} \mathcal{A}_{i}$ 's). The global theory $\mathcal{T}$ is then equivalent to the conjunctive combination of the corresponding sub-theories (the $S \mathcal{T}_{i}$ 's), where each sub-theory is the result of applying $\mathcal{C}$ to a constituent axiom set: $\mathcal{S} \mathcal{T}_{i} \stackrel{\text { def }}{=} \mathcal{C}\left(\mathcal{S} \mathcal{A}_{i}\right)$.

In CLD, we define $\mathcal{T}$ to be the result of conjunctive combination when $T$ is $C n\left(\bigcup_{i=1, \ldots, n} \mathcal{S} T_{i}\right)$; where $\mathrm{Cn}$ is the monotonic consequence (theory) operator in classical logic. When the corresponding axiom sets are understood, we will say that the global theory is conjunctively decomposable into these slice sub-theories. 11

In terms of the circumscriptions, we have:

$$
\mathcal{P} \mathcal{D C}(\mathcal{A}) \equiv \bigwedge_{i=1, \ldots, n} \mathcal{P} \mathcal{D C}\left(\mathcal{S} \mathcal{A}_{i}\right)
$$

Again, when the corresponding axiom sets are understood, we will also speak of a circumscription being conjunctively decomposable into slice circumscriptions, e.g., for $n=2$ :

$$
\begin{array}{r}
P D C(B ; D ; R ; Z) \equiv \\
P D C(S B 1 ; S D 1 ; S R 1 ; Z) \wedge \\
P D C(S B 2 ; S D 2 ; S R 2 ; Z)
\end{array}
$$

Figure 1 illustrates conjunctive decomposition with a flow diagram.

Conjunctive decomposition is thus a kind of reformulation or representation change. The global axiom

\footnotetext{
${ }^{11}$ Serial combination has the flavor of a cascade: there is a series of phases of adding axioms and drawing conclusions, where the previous stage's conclusions are treated as for-sure. Many NM inference procedures can be described in this manner. Details about serial decomposition are omitted due to considerations of space and focus. See [Grosof, 1992b] for more.
}

set and theory $\langle A, T\rangle$ are transformed into a collection of constituent axiom sets and slice sub-theories:

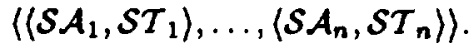

Most Subsets Do Not Qualify As Constituents for Decomposition: Note that, in general, in non-monotonic reasoning, one cannot blithely partition a global axiom set into a bunch of (distinct, or, more generally, overlapping) subsets (whose union is the global axiom set) any old way and get a conjunctive decomposition. This is because the axioms in one subset may conflict with those in another.

E.g., consider the classic Quaker-Republican example of conflict in default reasoning: there are two default axioms, one saying that Quakers are typically Pacifists, and another saying that Republicans are typically non-Pacifists. In addition, there are two for-sure axioms: that Nixon is a Quaker, and that he is a Republican. Suppose we consider two subsets: one containing the Quaker axioms, and another containing the Republican axioms. Treating a subset as a constituent axiom set means drawing non-monotonic conclusions from it as if there were no other axioms around. Doing so, from the first (with Quaker) one gets the default conclusion that Nixon is a Pacifist; from the second, one gets the default conclusion that Nixon is a nonPacifist. Taking the conjunction of these two "subtheories" thus results in garbage: inconsistency. Yet the actual global theory is consistent: neither conclusion about Pacifism is sanctioned. Figure 2 illustrates. 


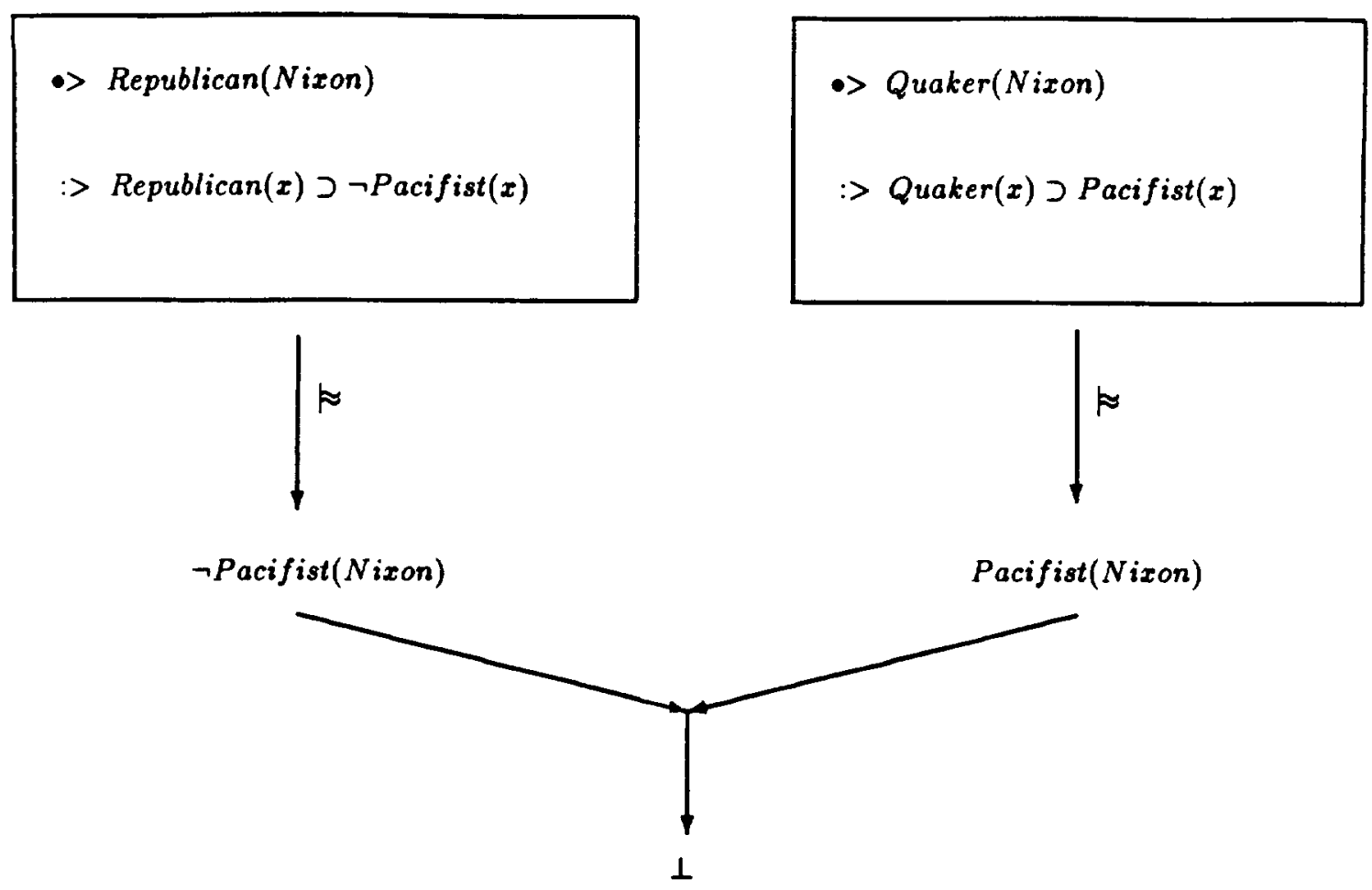

Figure 2: Non-modularity: Quakers and Republicans. (Default axiom labels not shown.)

Our spective is that in gena kind of logical non-modularity: when attempting to draw conclusions from a subset of the global axiom set, one must keep in mind the context of the remainder of the global axiom set. If one considers that remainder as an "internal" update, then that update may be non-monotonic. Another way to view this situation is that non-monotonicity means logical globality: in general, a non-monotonic conclusion cannot be drawn until the entirety of the global axiom set is considered.

\section{Locality:}

Suppose we can find a conjunctive decomposition in which for some $i$, the slice's axiom set is a subset of the global, i.e., $S \mathcal{A}_{i} \subseteq \mathcal{A}$. In this case, we say that the slice is a clean slice. Then we know that all the remaining axioms $\left(\mathcal{A}-\mathcal{S} \mathcal{A}_{i}\right)$ in the global axiom set are irrelevant context, in an important sense, relative to the slice's axiom set $\mathcal{S A}_{i}$. In this case, one can soundly, and in an important sense completely, perform inference locally: considering only the axioms in $S \mathcal{A}_{i}$, and using whatever standard procedures are available generally for the NM formalism. This is sound, because $\mathcal{C}\left(\mathcal{S} \mathcal{A}_{i}\right)$ is then a subset of the global theory. This is complete, in a sense, because the contribution of $\mathcal{S} \mathcal{A}_{i}$ to the global consequences requires only monotonic infer- ence beyond its own local (NM) consequences $\mathcal{C}\left(\mathcal{S} \mathcal{A}_{i}\right)$. By "irrelevant" above, then, we mean that one does not need to consider the remainder of the global axioms in order to do the essential non-monotonic aspect of the reasoning from $\mathcal{S A}_{i}$.

In the rest of this paper, we will be only considering decompositions that are clean. ([Grosof, 1992b], however, discusses the usefulness of decompositions that are not clean, e.g., decompositions on the basis of higher versus lower priority.)

Observe that in clean slicing, the constituent axioms sets are each smaller, and thus simpler, than the global axiom set. In prioritized default circumscription, and in other expressively rich NM formalisms, the computational complexity of non-monotonic reasoning (including, full forward inference and belief revision) is worse than monotonic reasoning. Non-monotonic reasoning (full forward inference and belief revision) in each slice, and vis monotonic conjunctive combination, is thus computationally less complex than non-monotonic reasoning in the global theory.

Partitioning Axioms As Kind of Reformulation: Our perspective, therefore, is that, in non-monotonic reasoning, decomposing, e.g., partitioning (see Theorems 1 and 12), g global axiom set into constituent axiom sets is a quite non-trivial kind of reformulation. This is very different from the situation in classical 
monotonic reasoning.

Safeties of Updating:

Suppose, in a conjunctive decomposition, that $\left\{\mathcal{S} \mathcal{A}_{1}, \ldots, \mathcal{S} \mathcal{A}_{k}\right\}$ are present both before and after an update $\mathcal{U}$. I.e., suppose that some of the constituent axiom sets in a decomposition after an update $\mathcal{U}$ are unchanged from (i.e., are the same as) in a decompositon before that update. Then we know that all of the conclusions in the conjunctive combination of their associated slices are safe under the update.

Hierarchy:

We can view the conjunctive combination of a set of slice sub-theories as being, in turn, a sub-theory. When those slices are clean, then this sub-theory is itself welldefined as a clean slice: its axiom set is simply the union of those slices'. Thus we can often choose grain size hierarchically during conjunctive decomposition.

Sequencing of Inference: See section 1 about concurrency.

\section{Disjoint Predicates}

Our results will all make use of the following idea of decomposing the specified prioritization.

\section{Composing Prioritization:}

The concept of prioritization over groups of defaults is natural in the specification process for many applications: often a group of defaults corresponds to a topic. [Grosof, 1991] introduced, and [Grosof, 1992b] elaborates, this idea of "composing" prioritization, in which an overall prioritization p.o. $R$ over the domain of individual defaults is equivalent to the result of composing an external prioritization p.o. $R E$, defined over groups, with a tuple $R I$ of prioritization p.o.'s, one $(R I i)$ per group, that each represent the prioritization internal to that group: $R=R E * R I$. Groups may, in turn, be composed of groups. Thus we may define prioritizations of prioritizations, in hierarchical or recursive fashion. Our example displays this structure.

Our first result is about decomposition on the basis of syntactic disjointness of predicates. It captures a basic case of the intuition that syntactically "having nothing to do with each other" should imply strong irrelevance of the kind we discussed in the last section.

Theorem 1

(Clean Decomposition, given Disjoint Predicates)

Let $P D C(B ; D ; R ; Z)$ be a global PDC. Let $\{B 1[Z 1], \ldots, B k[Z k]\}$ be a partition of the base axioms $B[Z]$, and let $\{D 1[Z 1], \ldots, D k[Z k]\}$ be a partition of the default formulas $D[Z]$, where the predicate tuples $Z 1, \ldots, Z k$ are a (disjoint) partition of $Z$. I.e., in terms of CLD, let there be a partition, of the base and default axioms, where the predicates mentioned in each element of the partition are disjoint. If a certain condition ( 0 ) (see below) on the prioritization $R$ is satisfied, then

$$
\begin{aligned}
P D C(B ; D ; R ; Z) & \equiv \\
\bigwedge_{j=1}^{k} P D C(B j ; D j ; R I j ; Z) & P
\end{aligned}
$$

(Note that the $Z$ on the right-hand side can be equivalently replaced by $Z j$.) Condition ( 0 ) is defined as: either, $R$ is the composition of some prioritization $R E$ with the tuple $R I$ of the internal prioritizations of each partition; or, $R$ is layered (stratified). The composition condition for non-layered $R$ corresponds, intuitively, to a kind of a partitionability of the prioritization. Note the special case of empty $R$ satisfies (0).

Proof Overview: Surprisingly non-trivial. The essence is to use the ability to separate existential quantifiers in the right-hand-side part of the circumscription formula (cf. section 3 ). Non-layered prioritization makes this tricky: hence the prioritization conditions in the theorem.

In terms of CLD, Theorem 1 tells us that syntactic disjointness implies irrelevance in the sense that we diacussed in the last section; the decomposition by syntactic partition is a clean slicing.

Theorem 1 immediately yields a powerful result about inference.

Theorem 2

(Locality of Inference, given Disjoint Predicates)

In Theorem 1 , each slice $j$ is sound and complete, relative to the global theory, for inference over its corresponding sub-language (partition of the predicates). That sub-language consists of the formulas that mention only the predicates $Z j$. This locality holds both for forward inference, and for backward inference (query-answering). Note that to perform inference using any subset $Y$ of the predicates $Z$, one need only work in the conjunctive combination of those slices whose predicates cover that subset $Y$.

Theorem 1 also immediately yields a powerful result about belief revision.

Theorem 3

(Safety of Updating, given Disjoint SubLanguages)

In CLD, let the previous axiom set be partitionable according to Theorem 1 . Let an update $U$ consist of base, default, and prioritization axioms, such that the formula parts of the base and default axioms mention only predicates from a (possibly empty) subset of the previous partitions, and such that the global prioritization condition ( 0$)$ is still met. Then all of the previous conclusions derived solely from the rest of the partitions' slices do not require retraction.

\section{Application to Main Example:}

The above theorems capture the first intuition that we discussed in section 2. At each point in the sequence of updates, Theorem 2 implies that inference can be localized: inferences about legged-ness can be performed in the slice that contains only the axioms about leggedness, and likewise for meetings. Figure 3 illustrates the 
original representation

conjunctive decomposition

legged-ness

meetings

$\mathrm{U} 1, \mathrm{U} 2, \mathrm{U} 4$

U3, U5 -- U8

Figure 3: Conjunctive Decomposition using Disjoint Predicates: In o"r main motivating example (section 2), we conjunctively decompose the global axiom set (after the last update $\mathrm{a}$. into two slices by employing the disjoint predicates result (Theorem 1): one slice about legged-ness, and the oth - slice about meetings. In the bottom half, each inner box stands for a constituent axiom set.

conjunctive decomposition of. Theorem 1 after the last update. Theorem 3 guarantees that after each meetings update, all of the previous conclusions drawn from the legged-ness slice are safe, and vice versa.

\section{Generalizations:}

Theorems 1, 2, and 3 generalize in several directions. Firstly, predicate (and function) symbols may overlap between the constituent axiom sets as long as they are fixed in the circumscription (see earlier discussion about fixing in section 3). Intuitively, it is OK to specify some predicate (and function) symbols as fixed if it is OK not to infer any default conclusions expressible purely in terms of those symbols. Secondly, the prioritization condition can be relaxed somewhat.

\section{Definitional Reformulation of Axioms:}

Thirdly, and mos! :nterestingly (see discussion toward end of section 1 about source of power), one can decompose with irrelevance (slice cleanly) as long as one can definitionally reformulate the global axiom set to meet the partitionability condition. (See Theorem 12.) One interesting such case is reasoning about one individual object, e.g., Joe in our example, at a time. (See Theorem 16.) Often (e.g., for the legged-ness axioms in our example), such re-formulability is easily (time polynomial in the number of axioms) detectable syntactically. We pursue all this in the next two sections.

\section{Basic Definitional Reformulation of Axioms, One-by-One}

Next, we define a particular kind of definitional reformulation. This kind of reformulation maps each formula in one formulation into a correspondent formula in another formulation, while preserving equivalence, i.e., without loss of information. Our motivation for considering this limited kind of reformulation is our intended application: to disjoint describability and its asocial-monadic special case. Why do we do the reformulation "one axiom at a time", i.e., one-by-one? Much of the reason is that there is an important difference between default / NM reasoning and monotonic reasoning.

We take for granted in monotonic logics that a collection of for-sure (base) axioms $B 1, \ldots, B m$ can be equivalently replaced by the axiom $B 1 \wedge \ldots \wedge B m$. In prioritized default circumscription and most other expressively rich NM formalisms, however, one cannot, in general, equivalently replace the pair of defat? axioms (whose default formulas are) $D 1$ and $D$ : the default axiom (whose default formula is the junction) $D 1 \wedge D 2$ (even in the case without prior: . i.e., when the prioritization is empty). Informati na. "grain size" of the defaults is important: having the two separate defaults means that, for example, $D 2$ may "succeed" (i.e., be concluded non-monotonically from the defaults) even if $D 1$ is "defeated" (e.g., is violated by the for-sure information), unlike if the only default present is $D 1 \wedge D 2$. We will need equivalencepreserving (and information-preserving) reformulation in order to apply the decomposition on the reformulated representation back onto the original representation.

Circumscription is defined in terms of second-order logic. We thus find it convenient and natural to define the kind of definitional reformulation we will need in terms of second-order logic, as well. We build on : : standard idea of a conservative extension, drawn t:the classical logical literature. In this and the next tion, we then develop several, increasingly complex tions of definitional reformulations, in order to har,ule 
the grouping structure in various stages of our reformulations: groups of predicates, groups of individuals, groups of formulas.

In this paper, we mainly address reformulations oriented around disjointness of mentioned (predicate and function) symbols. It is thus convenient to define our changes of representation in terms of changes in the symbols mentioned.

\section{First Cut at Definitional Reformulation:}

What does it mean to definitionally reformulate theories (or formulas) while preserving equivalence? At first glance, it simply means to introduce some definitions (of new symbols) which logically imply (entail) the equivalence of a theory expressed in an original set of symbols (i.e., an original representation) to a new theory expressed in those new symbols (i.e., a new representation). E.g., let $A 1[P]$ be the original theory, let $U[P, Q]$ be some definitions of new symbols $Q$ in terms of the old symbols $P$, e.g., a conjunction of explicit definitions:

$$
\begin{aligned}
U[P] & \stackrel{\text { def }}{\equiv} \\
(Q 1 & \equiv E 1[P]) \wedge \ldots \wedge(Q m \equiv E m[P])
\end{aligned}
$$

(where $m$ is the length of the tuple $Q$ ), and let $A 2[Q]$ be a new theory that is equivalent to $A 1[P]$ given $U[P, Q]$ :

$$
U[P, Q] \vDash A 1[P] \equiv A 2[Q]
$$

More generally, we can permit the new representation to use some of the old symbols; let $W$ be the overlap symbols between the old and the new. Suppose $A 1[W, Y]$ is the original theory, $A 2[W, Y, Z]$ is the new theory, and $U[W, Y, Z]$ is the (conjunction of) definitions of new symbols $Z$ in terms of $W$ and $Y$, e.g.,

$U[W, Y, Z] \stackrel{\text { def }}{\equiv}$

$$
(Z 1 \equiv E 1[W, Y]) \wedge \ldots \wedge(Z m \equiv E m[W, Y])
$$

(where $m$ is the length of the tuple $Z$ ); and suppose

$$
U[W, Y, Z] \vDash A 1[W, Y] \equiv A 2[W, Z]
$$

Then we call $U$ a "putative" definitional reformulator.

\section{Observation 4}

(Subtlety: Uninformativeness and Consistency) However, there is a subtlety. To us, part of the intuition behind the idea of an definitional reformulation is that the equivalence is non-spurious, i.e., that the definitions themselves are not introducing information. Unfortunately, merely requiring $U$ to be a conjunction of explicit definitions allows spuriosity and informativeness.

Consider the following example. Let $W$ be empty. Let $Y \stackrel{\text { def }}{=}\langle Y 1, Y 2\rangle$, where $Y 1$ and $Y 2$ are 0 -ary predicates. ${ }^{12}$ Let $A 1[W, Y]$ be defined as $Y 1 \wedge \neg Y 1$. Let the definitions $U$ be $(Z 1 \equiv Y 1) \wedge(Z 2 \equiv \neg Y 1)$, where $Z 1$ and $Z 2$ are 0 -ary predicates. Let $Z \stackrel{\text { def }}{=}\langle Z 1, Z 2\rangle$, and let $A 2[W, Z]$ be defined as

\footnotetext{
${ }^{12}$ We do not use $Y 2$ immediately, but we will use it later when we continue this example in the discussion after Definition 7 .
}

$Z 1 \wedge Z 2$. Then $U$ implies that $A 1$ is equivalent to $A 2$. Yet this contravenes our intuition of a reasonable definitional reformulation. $A 1$ is inconsistent, i.e., is equivalent to False. A2, by contrast, is consistent.

Viewing the direction of reformulation from $A 2$ to $A 1$, in effect $U$ is introducing some information, namely that $Z 1 \equiv \neg Z 2$. The source of this problem is that, even though $U$ is a conjunction of explicit definitions, $U$ is itself not always consistent when it is viewed in this "return direction" of the reformulation (i.e., from $A 2$ to $A 1$ ). Yet, to us, any notion of equivalencepreserving definitional reformulation ought to be symmetric, i.e., kosher in both directions: from $A 1$ to $A 2$ and from $A 2$ to $A 1$. We would, therefore, like to impose some kind of additional constraint on $U$ to guarantee intuitive uninformativeness and non-spuriosity of the equivalence between the two representations. Below, we do this by formalizing $U$ 's consistency and its relationship to directionality more precisely.

The idea of a conservative extension, standard in the classical logical literature, provides a nice notion of uninformativeness in terms of mentioned symbols.

\section{Definition 5 (Conservative Extension)}

Let $A 1[P]$ be a formula ${ }^{13}$ mentioning only (the tuple of symbols) $P$. Let $Q$ be (a tuple of symbols) distinct from $P$. Let $A 2[P, Q]$ be a formula mentioning only $P \cup Q$. Then we say that $A 2[P, Q]$ is a conservative extension of $A 1[P]$ when:

$\forall P .[(\exists Q . A 2[P, Q]) \equiv A 1[P]]$

or, equivalently, when both:

$$
\begin{aligned}
& \forall P, Q \cdot A 2[P, Q] \supset A 1[P] \\
& \forall P \cdot[A 1[P] \supset(\exists Q \cdot A 2[P, Q])]
\end{aligned}
$$

Another way to view the idea of conservatism in this definition is that $A 2$ "says" exactly as much about $P$ as $A 1$ does. $A 2$ in addition says stuff about $Q$. I.e., for any formula $G[P]$ mentioning only $P$ :

Suppose that

$$
A 2[P, Q] \vDash G[P] \quad \Longleftrightarrow \quad A 1[P] \vDash G[P]
$$

$$
A 2[P, Q] \stackrel{\text { def }}{\equiv} A 1[P] \wedge U[P, Q]
$$

Then we say that $U[P, Q]$ is a conservatively extending update to $A 1[P]$.

Intuitively, we can thus view a conservatively extending update $U[P, Q]$ as uninformative in a precise sense, namely about the old symbols $P$.

\section{Notation:}

Let $D \leq E$ stand for the universally quantified implication $\forall x . D(x) \supset E(x)$, where $D$ and $E$ are open formulas with the same arity of free variables (i.e., are similar), and $x$ stands for a tuple of free individual (object) variables. Let $D=E$ then be defined analogously as the universally quantified equivalence $\forall x . D(x) \equiv E(x)$. We also apply this notation to tuples $D=\left(D_{1}, \ldots, D_{m}\right\rangle$ and $E=\left\langle E_{1}, \ldots, E_{m}\right)$ : e.g.,

\footnotetext{
${ }^{13}$ in (higher-order) classical logic
} 
$D=E$ stands for

$$
D_{1}=E_{1} \wedge \ldots \wedge D_{m}=E_{m} \text {. }
$$

Fact 6

(Explicit Definitions Are Conservative)

(Conjunctions of) explicit definitions of new symbols (e.g., predicates) are always conservatively extending updates. I.e., in Definition 5, suppose $U[P, Q]$ is a conjunction of explicit definitions of each symbol in $Q$ : $Q=E[P]$

(Here, we are using the tuple $=$ notation introduced above, and applying it also to functions and terms.) Then $U[P, Q]$ is a conservatively extending update, for any $A 1[P]$.

Conservative Extension, Uninformativeness, and Directionality:

Equipped with the idea of a conservative extension, we are now ready to return to the question of refining the basic idea of definitional reformulation. In our "first cut" above, we found a need to formalize the constraint that the putative definitional reformulator $U$ be uninformative, in both directions of the reformulation. In Definition 5, we observed that the property that a "definitional" reformulator $U[P, Q]$ is a conservatively extending update precisely expresseses $U$ 's uninformativeness, in the direction of $A 1$ to $A 2$, i.e., about $P$. There, however, $U$ is not really quite a reformulator in the sense we discussed in the "first cut", since $A 2$ mentions not just the new symbols $Q$, but also the old symbols $P$. However, we can extract the notion of uninformativeness present there, i.e., the "conservatism" in the idea of a conservative extension.

The property that $U$ is a conservatively extending update is: $\quad A 1[P] \vDash \exists Q . U[P, Q]$

which we can also write as:

$$
\vDash(\forall P . A 1[P] \supset \exists Q . U[P, Q])
$$

One can view the right-hand-side as a satisfiability (i.e., consistency) property. This satifiability / consistency is conditional on $A 1$.

We take this conservativeness property as the basis for uninformativeness of a (putative) definitional reformulator $U$. However, we need the "return direction" uninformativeness as well:

$A 2[Q] \vDash \exists P . U[P, Q]$

which we can also write as:

$$
\vDash(\forall Q . A 2[Q] \supset \exists P . U[P, Q])
$$

\section{Definition 7}

\section{(Definitional Reformulator - Basic Case)}

We say that $U[W, Y, Z]$ is a definitional reformulator (basic case) between two formulas $A 1[W, Y]$ and $A 2[W, Z]$ (where $W, Y$, and $Z$ are distinct tuples of symbols) when:

1. $U$ implies the equivalence of $A 1$ and $A 2$ :

$$
\vDash U[W, Y, Z] \supset(A 1[W, Y] \equiv A 2[W, Z])
$$

2. $U$ is uninformative, i.e., conservative, in both directions of the reformulation, i.e., with respect to $A 1$ and with respect to $A 2$ :

$$
\begin{array}{lll}
\vDash & (\forall W, Y . A 1[W, Y] \supset \exists Z . U[W, Y, Z]) \\
=(\forall W, Z . A 2[W, Z] \supset & \supset Y . U[W, Y, Z])
\end{array}
$$

Discussion; Directionality:

Having the second direction, in addition to the first direction, of the conservativeness property in Definition 7 rules out the nastily-behaved example that we discussed in Observation 4. However, the conservativeness property in Definition 7 reassuringly does permit, for example, the following, more intuitively reasonable basic-case definitional reformulator:

$$
U[W, Y, Z] \stackrel{\text { def }}{\equiv}(Z 1 \equiv Y 1) \wedge(Z 2 \equiv \neg Y 2)
$$

(where the symbols are as in the example discussed in Observation 4) for any $A 1, A 2$.

The property that $U$ consists exclusively of (a conjunction of) explicit definitions ensures, in general, only one direction of conservativeness.

\section{Conditionality Versus Unconditionality of Con-} servativeness:

Definition 7 is perhaps too "custom" in one regard, however. The conservativeness property is conditional: it depends on the particular $A 1$ and $A 2$. This is perhaps unsatisfactory intuitively, at least for some purposes, as a notion of "definitional" in "definitional reformulator".

Alternative Definition of Conservativeness: Unconditional Version:

As an alternative definition of the basic case of definitional reformulator, we observe that one can use a stronger (i.e., more strongly constrained, special case) notion of conservativeness instead:

$$
\begin{aligned}
& =\forall W, Y . \exists Z . U[W, Y, Z] \\
& =\forall W, Z . \exists Y . U[W, Y, Z]
\end{aligned}
$$

to replace the conservativeness property (2.) in Definition 7. This "unconditional" version of the conservativness property does not depend on $A 1$ and $A 2$ : i.e., it implies that the "conditional" conservativeness property (2.) in Definition 7 holds for any $A 1$ and $A 2$.

\section{Alternative Definition of Conservativeness: Backgrounded Version:}

As an intermediate position between the conditional and unconditional versions of the conservativeness property, we observe that one can formulate conditionality in a somewhat abstracted fashion: in terms of the symbols $W$ that are in common between the two representations. We will find it convenient for our later definitions to employ a notion of a background $G[W]$ to the reformulation. One can view $G[W]$ as, in effect, included in both $A 1[W, Y]$ and $A 2[W, Z]$. We then define the "backgrounded" version "f the conservativeness property as:

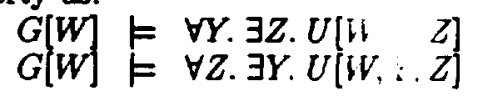


In the remainder of this paper, we will use this last, "backgrounded" version of the conservativeness property. We do so in order to formally simplify our later definitions of more complex kinds of definitional reformulators and reformulations, which are oriented towards particular uses. However, the "conditional" version of the conservativeness property is more fundamental and general, we believe, and is interesting to explore: we plan to do so in the future.

\section{No Requirement of Explicitness:}

Note that in Definition 7 , we did not require $U$ to be in the form of a conjunction of explicit definitions of new symbols in terms of old symbols. We formalized / summarized the "definitional" flavor of the reformulator as, simply, its conservativeness. Our definition of definitional reformulator thus allows $U$ to consist of implicit definitions (e.g., with recursion) and partial definitions (i.e., necessary and sufficient conditions). (Later, in our result about the asocial monadic special case of disjoint describability (Theorem 16), the reformulator will consist exclusively of explicit definitions, however.)

Next, we define a definitional reformulation of a group of formulas, using a single common reformulator: one-by-one, into a new group of formulas. For this purpose, it is convenient to be able to abstract away from conditionalizing conservativeness on each of those formulas: we thus use the backgrounded version of conservativeness.

\section{Definition 8 (Group Reformulator)}

Let $E T 1[W, Y]$ and $E T 2[W, Z]$ each be a similar ${ }^{14}$ tuple of formulas; these formulas may be open or closed. We call each tuple a group. Let $U[W, Y, Z]$ and $G[W]$ be closed formulas. We say that $U[W, Y, Z]$ is a group reformulator between $E T 1[W, Y]$ and $E T 2[W, Z]$, given the background $G[W]$ when:

1. $U$ is conservative (given the background) with respect to $Y$ and also with respect to $Z$ :

$$
\begin{aligned}
& G[W] \vDash \forall Y . \exists Z . U[W, Y, Z] \\
& G[W] \vDash \forall Z . \exists Y . U[W, Y, Z]
\end{aligned}
$$

2. $U$ reformulates each formula in either group into the corresponding formula in the other group. I.e., $U$ implies the equivalence of corresponding member formulas (subscripted by $j$ ) in the two groups:

$$
\begin{aligned}
U[W, Y, Z] \wedge & \underset{G}{\forall}[W] \underset{E T}{ } \vDash j[W, Y]=E T 2 j[W, Z]
\end{aligned}
$$

\section{Disjoint Describability and Disjoint Individuals}

Next, we show how to use definitional reformulation to generalize the disjoint predicate special case: to the more general case of disjoint describability. More precisely, we use definitional reformulation to transform

\footnotetext{
${ }^{14}$ Terminology: By "similar", we mean of same length, and with same arities for their members.
}

a disjointly describable global axiom set into a representation that has disjoint predicates, and then to transform back again after decomposition. Figure 4 illustrates. We show that the disjoint describability case, like disjoint predicate case, has a clean, partitioning conjunctive decomposition, which, moreover, implies interesting localities of inference and safeties of updating. We then identify an interesting special case of disjoint describability (asocial-monadic) that, like the disjoint predicate case, is easily recognizable in terms of the syntax of the starting global axiom set.

We begin with some preliminaries.

Definition 9 (Syndicate Reformulator)

We define a syndicate reformulator as a tuple of group reformulators that obeys an extra syndication property: their conjunction is also conservative.

More precisely: Let $E T T 1[W, Y]$ and $E T T 2[W, Z]$ each be a similar tuple of tuples of formulas; these formulas may be open or closed. Each element of the top level of tupling is itself a tuple of formulas cf. Definition 8. The top level tuple is thus a syndicate whose elements are groups of formulas.

Let $U T[W, Y, Z]$ be a tuple of closed formulas, of the same length as the top level tuples above. I.e., let it consist of one formula per group. Let $G[W]$ be a closed (background) formula, as in Definition 8.

Below, we use $i$ to subscript groups, and $j$ to subscript formulas within groups.

We say that $U T[W, Y, Z]$ is a syndicate reformulator between $E T T 1[W, Y]$ and $E T T 2[W, Z]$, given the background $G[W]$ when:

1. For each group $i, U T i$ is a group reformulator between ETTi and ETT2i (given the background):

$$
\begin{aligned}
\forall i . U T i[W, Y, Z] \wedge G[W]= \\
\forall j . E T T 1_{i j}[W, Y]=E T T 2 i j[W, Z]
\end{aligned}
$$

2. the conjunction $U C \stackrel{\text { def }}{\equiv} \bigwedge_{i} U T i$ is conservative (given the background) with respect to $Y$ and also with respect to $Z$ :

$$
\begin{aligned}
& G[W] \vDash \forall Y . \exists Z . U C[W, Y, Z] \\
& G[W] \vDash \forall Z . \exists Y . U C[W, Y, Z]
\end{aligned}
$$

The reason we call the above a syndicate reformulation is the linkage between the different groups imposed by the conjunction's ( $U C$ 's) conservative extension property. This implies, but is not implied by, the conjunction of the conservative extension properties for each group's reformulator $U T i$.

\section{Definition 10}

(Partitioning Syndicate Reformulator)

We say that a syndicate reformulator of. Definition 9 is $Z$-partitioning when:

$$
\begin{aligned}
& \forall i . U T i[W, Y, Z] \stackrel{\text { def }}{\equiv} U i[W, Y, Z i] \\
& \forall i, j . E T T 2 i j[W, Z] \stackrel{\text { def }}{\equiv} E T T 2 i j[W, Z i]
\end{aligned}
$$

where $\forall j \neq k . Z j \cap Z \bar{k}=\bar{\emptyset}$, i.e., the appearances of the symbols $Z$ are partitioned by group. 


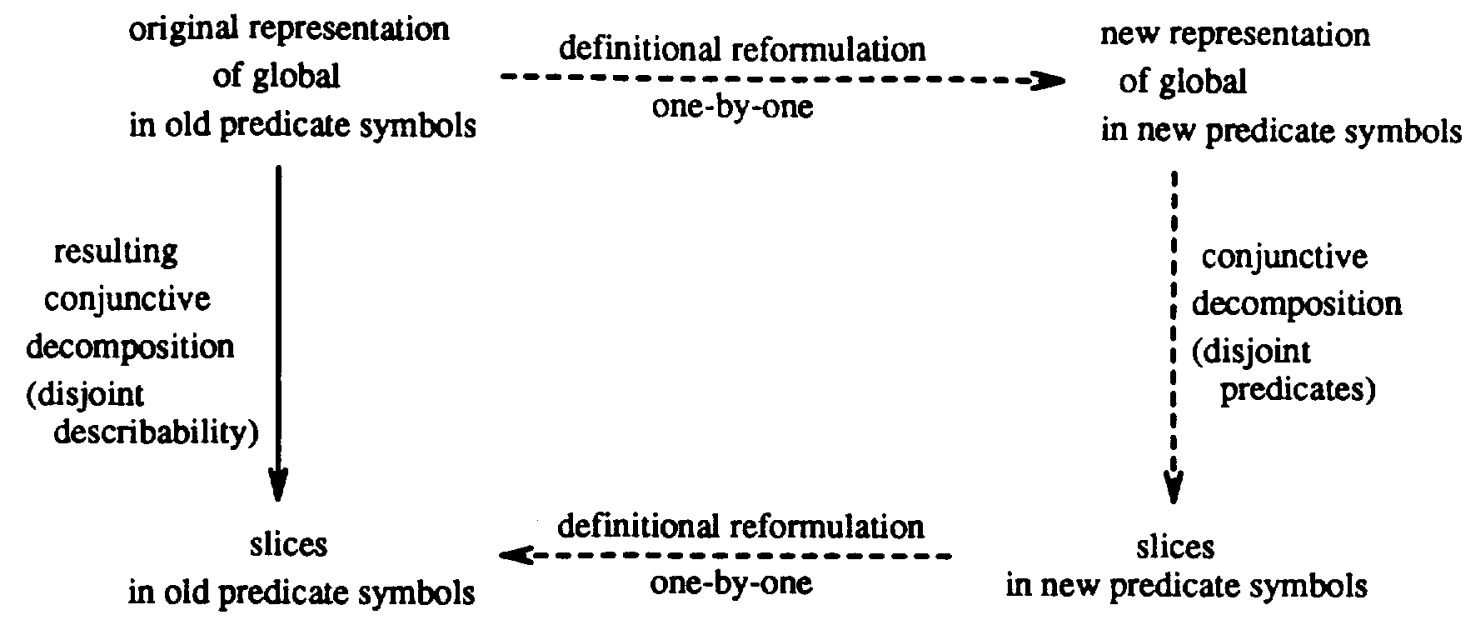

Figure 4: Disjoint Describability: a inw diagram of the reformulation steps involved.

Definition 11 (Disjoint Describability)

Suppose that $U T$ is a $Z$-partitioning syndicate relormulator as in Definition 10, where for each group $i$, ETT $1 i$ is defined as the concatenation of a (closed, base) formula $B 1 i$ with a tuple of (open, default) formulas $D 1 i$, and similarly, ETT2 $i$ is the concatenation of $B 2 i$ and $D 2 i$.

Let $B 1$ stand for the conjunction of the $B 1 i$ 's; and $D 1$ stand for the concatenation of the $D 1 i$ 's. Let $B 2$ and $D 2$ be defined similarly.

Suppose also that $P D C(B 2 ; D 2 ; R ;$ fix $W ; W, Z)$ fulfills the conditions in Theorem 1 (disjoint predicates), where the grouping, and the partition there on $Z$, is the same as in $U T$.

Then we say that $P D C(B 1 ; D 1 ; R ;$ fix $W ; W, Y)$ is disiointly describable under (definitional) reformulat. . by $U T[W, Y, Z]$, given $G[W]$.

\section{Theorem 12}

(Clean Decomposition, given Disjoint Describability)

If a PDC is disjointly describable, then it is cleanly conjunctively decomposable into slices corresponding to the partitioning grouping employed in the reformulation. I.e., then the grouping employed in the reformulation forms the basis for a clean slicing.

More precisely: Suppose

$P D C(B 1 ; D 1 ; R ;$ fix $W ; W, Y)$ is disjointly describable under (definitional) reformulation by $U T[W, Y, Z]$, given $G[W]$, as in Definition 11. Then

$$
\begin{aligned}
& P D C(B 1 ; D 1 ; R ; f i x W ; W, Y) \equiv \\
& \bigwedge_{i} P D C(B 1 i ; D 1 ; R i ; f i x W ; W, Z i)
\end{aligned}
$$

where $R i \stackrel{\text { def }}{=} R^{N i}$ is the internal prioritization of the group of defaults $D 1 i$, whose index set (tuple) is $\mathrm{Ni}$. (Equivalently, the $Z i$ on the right hand side could be replaced by $Z$.)

Proof Overview: Theorem 1 plus some lemmas about definitional reformulation of circumscriptions.
Figure 4 illustrates the logical flow of the proof.

Theorem 12 immediately yields results about locality of inference, using Theorem 2, and about safety of updating, using Theorem 3 .

Next, we consider a special case of disjoint describability: asocial-monadic.

\section{Theorem 13}

(Fixed Cases Reformulation of Defaults)

In PDC, defaults can be reformulated by relativizing them to fixed (-formula) cases.

More precisely: In a $P D C\left(B ; D^{N} ; R ; f i x \quad W ; Z\right)$, suppose that

$$
\forall i \in N . B[Z] \vDash \forall x i . V_{j=1}^{m i} F i j[Z, x i]
$$

where $x i$ is a (possibly empty) tuple of individual (object) variables, and where, for each $i, j$, the (possibly) open (elementary) formula $F i j[Z, x i]$ is fixed relative to the circumscription (e.g., it mentions only function symbols; remember all functions are fixed). For each default index $i$, we call each $F i j$ a fixed case. Suppose also that

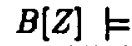

$$
\forall i, j . \forall x i . E i j[Z, x i] \equiv(F i j[Z, x i] \supset D i[Z, x i])
$$

I.e., suppose that each $E i j$ is equivalent to the default $D i$ relativized to the fixed case $F i j$. Then

$$
\begin{gathered}
P D C(B ; D ; R ; f i x W ; Z) \equiv \\
P D C(B ; E ; R R ; f i x W ; Z)
\end{gathered}
$$

where the tuple $E$ stands for the concatenation of all the $E_{i j}$ 's, and where $R R$ is defined as the composition of $R$ (as external prioritization) with a tuple $\theta T$ of empty prioritization p.o.'s. Each of $\theta T$ 's elements is an empty prioritization p.o. OT $i$ that is of size $m$ and corresponds to (i.e., has as domain) the index se of the (sub-) tuple $E i$.

Proof Overview: The key is that each origina default pre-order is equivalently reformulated, in the 
context of the circumscription's "augmentation" (i.e., second-order-quantified part in its definition cf. section 3 ), into a parallel default pre-order corresponding to Ei. ㅁ

Definition 14 (Asocially Monadic)

We say that a prioritized default circumscription $P D C(B ; D ; R ;$ fix $W ; Z)$, or a corresponding CLD axiom set, is asocially monadic when:

1. All predicates in $Z$ are monadic, i.e., 1-ary (a.k.a., unary).

2. The base sentence $B$ has the form of a conjunction of universal ${ }^{15}$ formulas. We will refer to these as the base formulas (axioms).

3. Every default formula (axiom) in $D$ is quantifier-free.

4. No base sentence (axiom) in $B$, and no default formula (axiom) in $D$, "mixes" individuals. I.e., in their clausal forms, no clause contains two literals with different arguments. Intuition: different individuals "don't want to have anything to do with each other", i.e., they are "asocial".

5. All terms appearing in the base and default formulas are ground, except for primitive variables.

6. The prioritization $R$ is either layered (e.g., parallel), or it is point-modular (see definition below).

7. All (explicit) fixtures are of predicate symbols $(W)$, rather than of arbitrary formulas. (In addition, as usual, all function symbols are fixed.)

8. Uniqueness of Names Axioms (UNA): The base $B$ includes axioms enforcing the distinctness of all terms that appear in the base and default axioms.

9. Besides in the UNA, equality does not appear in the base or default formulas. (Remember, equality, when viewed as a predicate, is binary, not monadic.)

\section{Definition 15}

\section{(Point-Modular Prioritization)}

Point-modular prioritization generalizes (i.e., the class includes) the prioritization that is typical in default inheritance networks. By "point" here, we mean an individual in the logical language, either named (a ground term, e.g. $E d$ ) or unnamed (e.g., referred to by a first-order variable, e.g., $x$ in $\operatorname{bat}(x) \supset 2 \operatorname{legs}(x)$ ). (This idea of a point can be straightforwardly generalized to a tuple of individuals (e.g., $(B o s s(4321), d\rangle)$ to handle predicates / formulas with arity more than one; but we are only considering here the unary case in the context of the asocially monadic case.) By pointmodular, we mean that the overall prioritization is equivalent to the composition of some external prioritization (over the points) composed with a tuple of internal prioritizations, one per point. Point-modularity results when the prioritization is only specified between the same instantiations of different defaults.

\footnotetext{
${ }^{15}$ Terminology: By universal, we mean without existential quantifiers.
}

E.g., when the bat default has higher priority than the mammal default at each point: (the default axiom whose default formula is) bat (Betsy) つ 2legs (Betsy) takes precedence over (the default axiom whose default formula is) mammal(Betsy) つ 4legs(Betsy), bat (Joe) つ $2 \operatorname{legs}(J \circ e)$

takes precedence over mammal(Joe) つ4legs(Joe), bat $(F i d o) \supset 2 \operatorname{legs}(F i d o)$ takes precedence over mammal(Betsy) つ 4legs(Betsy), etc., but there is no precedence between the defaults at different points, e.g., between bat(Betsy) つ 2legs(Betsy) and mammal(Joe) $\supset$ 4legs (Joe). Unfortunately, we do not have space to define point-modularity in further detail here; it requires discussing "pointwise" prioritization somewhat similar to that in [Lifschitz, 1988], and generalizing CLD to increase its expressivity with respect to prioritization. Note, however, that many point-modular prioritizations can be expressed in CLD. See [Grosof, 1992a] for more.

\section{Theorem 16}

(Decomposition by Reformulation, IndividualWise)

Suppose the $P D C(B 0 ; D 0 ; R 0 ; f i x W ; Z)$ is asocially monadic cf. Definition 14. Then the circumscription can be cleanly sliced, i.e., conjunctively decomposed, into its individual-wise reformulation:

$P D C(B 0 ; D 0 ; R 0 ;$ fix $W ; Z) \equiv$

$$
\bigwedge_{j=1}^{m+1} P D C(B 1 j ; D 1 j ; R j ; \text { fix } W ; Z)
$$

This individual-wise reformulation is defined as follows.

The basic idea of the reformulation is to divide the base and default axioms into groups: one group per named individual, plus a catch-all "remainder" group for all other, unnamed individuals. Some reformulation, of a relatively simple kind that is different from decomposition and one-by-one definitional reformulation, is involved in order to break up the quantified base axioms and the open defaults into these cases. Figure 5 illustrates this logical flow. The details of the overall reformulation are, however, a bit involved to define; bear with us.

To begin with, we partition the base and default formulas according to which arguments appear in them.

Let $J \stackrel{\text { def }}{=}\{1, \ldots, m\}$ index the set of all ground terms $a j$ that appear in the base or default formulas.

Let $B 0 j$ stand for the tuple of base formulas that mention $a j$. Each of its members we write as $B 0 j k[Z]$.

Let $B O V$ stand for the tuple of base formulas, other than the UNA, that mention a free variable (all of these are universally quantified). Each of its members we write as $\forall x . B 0 V k[Z, x]$. Here $x$ is a single (free) individual variable.

We treat the default formulas similarly to the base. Let $D 0 j$ stand for the tuple of default formulas that mention $a j$. Each of its members we write as $D 0 j k[Z]$.

Let $D 0 \mathrm{~V}$ stand for the tuple of default formulas that mention a free variable (i.e., that are open; all of these 


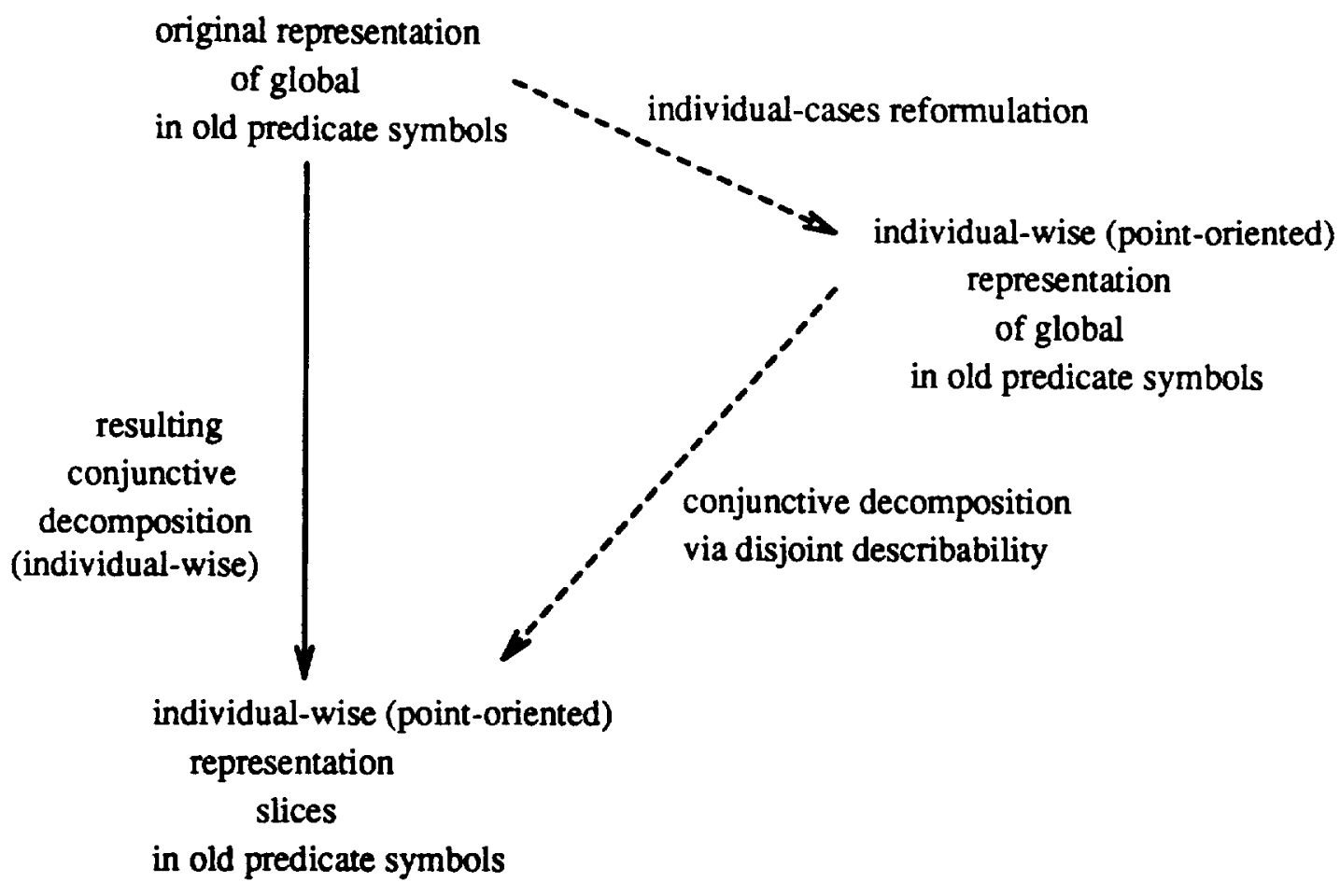

Figure 5: Asocially Monadic: a flow diagram of the reformulation steps involved. See also Figure 4.

are quantifier-free). Each of its members we write as $\operatorname{DoVk}[Z, x]$. Here $x$ is a single (free) individual variable.

Next, we reformulate the base and default formulas that mention a variable.

For each $j \in J$, let $B 1 V j$ stand for the instantiation of the quantified base formulas $B 0 V$ to $a j$. Each of its members $B 1 V j k[Z]$ is defined as the formula $B 0 V k[Z, a j]$.

Let $U N N A M E D[x]$ stand for the formula $\bigwedge_{j \in J} \boldsymbol{x} \neq a j$.

Let $B 2 V$ stand for the tuple of quantified base formulas after relativization to the unnamed case. Each of its members $B 2 V k[Z]$ is defined as:

$$
\forall x . U N N A M E D[x] \supset B O V k[Z, x]
$$

For each $j \in J$, let $D 1 V j$ stand for the instantiation of open default formulas $D O V$ to $a j$. Each of its members $D 1 V j k[Z]$ is defined as the formula $D 0 V k[Z, a j]$.

Let $D 2 \mathrm{~V}$ stand for the tuple of open default formulas after relativization to the unnamed case. Each of its members $D 2 V k[Z]$ is defined as:

$$
U N N A M E D[x] \supset D O V k[Z, x]
$$

For each $j \in J$, Let $B 1 j$ stand for the conjunction of (all members of) the tuples $B 0 j$ and $B 1 V j$.

For $j=m+1$ (i.e., the unnamed case), let $B 1 m+1$ stand for the conjunction of (all members of) the tuple $B 2 V$ plus the UNA.

For each $j \in J$, Let $D 1 j$ stand for the concatenation of the tuples $D 0 j$ and $D 1 V j$.

For $j=m+1$ (i.e., the unnamed case), let $D 1 m+1$ stand for the tuple $D 2 \mathrm{~V}$.

Let $R j$ be defined as the prioritization internal to $D 1 j$, i.e., as $R^{N j}$, where, for each $j=1, \ldots, m+1, N j$ is the index tuple of $D 1 j$.

Proof Overview: We use a first stage of reformulation employing Theorem 13. This stage involves what we called above an "extra" kind of reformulation: e.g., to reformulate each open default axiom and each quantified base axiom into a collection of "point"case (individual-case) axioms, plus a remainder-case (unnamed case) axiom. Then we use a second stage partitioning syndicate reformulation into disjoint describability, employing Theorem 12 . In that second stage of reformulation, we treat the UNA as background. There, the newly introduced predicates are all 0 -ary, except for those corresponding to the catchall case. The definitional reformulator consists of the explicit definitions of these newly introduced predicates. There is one new predicate for each ground atom in the original representation. Note that the second stage itself combines two kinds of reformulation: definitional reformulation, to transform into a representation with disjoint predicates, and conjunctive decomposition.

Figure 5 illustrates the logical flow of the reformula- 
original representation

global

U1 -- U8

conjunctive decomposition $\rfloor$ disjoint predicates

\begin{tabular}{|cc|}
\hline legged-ness & meetings \\
\hline $\mathrm{U} 1, \mathrm{U} 2, \mathrm{U} 4$ & $\mathrm{U} 3, \mathrm{U} 5$-- U8 \\
\hline
\end{tabular}

\begin{tabular}{c|c} 
conjunctive \\
decomposition
\end{tabular} \mid $\begin{aligned} & \text { individual-wise (asocially monadic) } \\
& \text { applied to legged-ness slice }\end{aligned}$

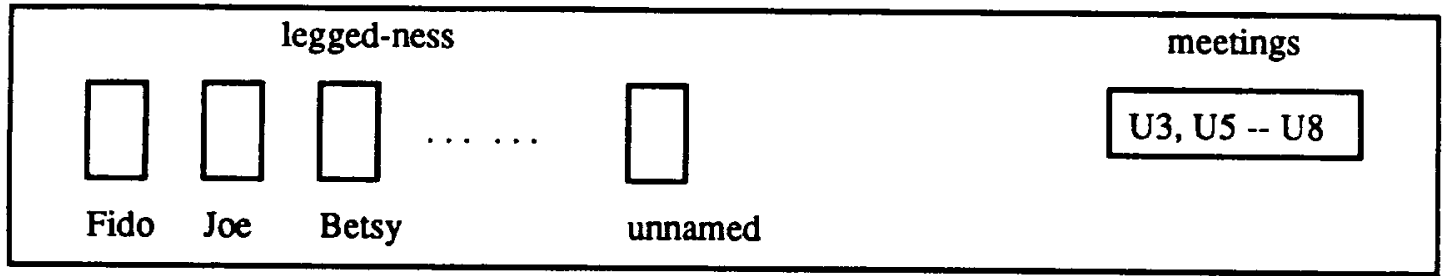

Figure 6: Conjunctive Decomposition using Asocially Monadic and Disjoint Predicates: In our main motivating example (section 2), we can conjunctively decompose the global axiom set (after the last update $U_{8}$ ) into two slices by employing the disjoint predicates result (Theorem 1): one slice about legged-ness, and the other slice about meetings. This first-stage decomposition is the same as in Figure 3. We can conjunctively decompose the legged-ness slice, individual-wise, by employing the asocially monadic result (Theorem 16). That is, in a second stage, we slice (more finely) within a slice that arose from the first stage. The second stage thus yields a second, finer-grain decomposition of the global axiom set, containing the meetings slice (unchanged from the first stage) plus each of the individual-case legged-ness slices. Together, the two stages exemplify the ability to decompose hierarchically / recursively. Each of the named-individual / "point" slices in the second stage contains a set of axioms that correspond to the instantiation / particularization of the original legged-ness axioms $\left(u_{1}, U_{2}\right.$, and $\left.U_{4}\right)$ to (the case of) one named individual, e.g., Joe. Each outer box stands for a decomposition. Each inner box stands for a constituent axiom set.

tion steps involved; it builds upon Figure 4.

Application to Main Example: (Continued from the discussion in section 5:) Consider our main motivating example (about legged-ness and meetings, from section 2). There, after the final update $\mathcal{U}_{8}$ (and, indeed, at any earlier point in the sequence of updates), the legged-ness slice, i.e., the set of axioms about legged-ness $\left(U_{1}, U_{2}\right.$, and $\left.U_{4}\right)$ is asocially monadic. It can thus be conjunctively decomposed cleanly, individual-wise. Figure 6 illustrates and explains this decomposition. As we discussed earlier, the definitional reformulation involved in the individualwise decomposition cf. Theorem 16 introduces a new 0 -ary predicate for each ground atom in the original representation; in this example, two such new predicates are:

$$
\begin{aligned}
& \text { nbatJoe } \equiv \text { bat (Joe) } \\
& \text { n2legsJoe } \equiv 2 \operatorname{legs}(\mathrm{Joe})
\end{aligned}
$$

Theorem 17

(Individual-wise Locality of Inference, when Asocially Monadic)

In Theorem 16, each slice $j$, where $j$ is the (index of) a named individual (cf. statement of that Theorem), is sound and complete, relative to the global theory, for inference over its corresponding sub-language. That sub-language consists of the ground formulas (sentences) in which the only individual mentioned is $j$ (e.g., Betsy). This locality holds both for forward inference, and for backward inference (query-answering). Note that to perform inference using any subset $S J$ of the named individuals $J$, one need only work in the conjunctive combination of those slices corresponding 
to $S J$

For query-answering about a new named individual $b$ (named in the query), just introduce the new term $b$ into the set of terms that are indexed by $J$ in the theorem. The only additional requirement is that the UNA ensure its distinctness from the other named individuals.

Application to Main Example: Thus after each update, inferences about any named individual's (e.g., Joe's) legged-ness can be made by working in a slice axiom set that has been instantiated / particularized to that individual $(\mathrm{J} o e)$. One advantage of this is that simpler inference algorithms are available for such an expressively simpler axiom set. In this case, there is a decidable polynomial-time procedure (see "totalpropositional" case results in [Grosof, 1992b]). By contrast, there is no general inference procedure, even for query-answering, yet available for the full example (i.e., including the meetings aspect). (See next section for discussion of inference procedures available for prioritized circumscription.) This illustrates that decomposition-type reformulation is useful to exploit available / known tractable special cases to do part of the inference in a NM theory, even when the overall theory is intractable or undecidable (see next section for more discussion of this point.)

Theorem 16 also immediately yields a powerful result about belief revision.

\section{Theorem 18}

- (Safety of Updating, when Asocially Monadic) In CLD, let the previous axiom set be asocially monadic. Let an update $u$ consist of base, default, and prioritization axioms, such that the formula parts of the base and default axioms are ground and mention only a set of named individuals $I U$. Then all of the previous conclusions terived solely from the rest of the named individuals $\quad s$ (i.e., the slices according to Theorem 16) are sal. ..der the update.

\section{Application to Main Example:}

E.g., after update $\mathcal{U}_{4}$ (mentioning only Joe and Spot), this theorem tells us that we do not have to re-consider whether the previous conclusion 2legs(Betsy) is still sanctioned: it must be preserved. Thus we can know, with relatively little computational work (see discussion of complexity in next section), that most of the previous $\mathbf{N M}$ conclusions are safe.

\section{Disjoint Groups of Individuals:}

Definition 14 and Theorems 16, 17, and 18 also generalize straightforwardly to considering disjoint groups of individuals, where any syntactic mixing in the axioms involves only individuals within the same group.

\section{Discussion, Conclusions, and Future Work}

Proof Procedures: Prioritized default circumscription is expressively reducible to prioritized predicate circumscription (see section 3 ). There exist several backward proof procedures for fairly expressive classes of prioritized predicate circumscription, including for layered (stratified) prioritization [Przymusinski, 1989] [Ginsberg, 1989] [Baker and Ginsberg, 1989] [Inoue and Helft, 1990] [Inoue et al., 1991]. More interestingly, [Geffner, 1989] contains a proof theory and proof procedures which promise to be easily adaptable (using an equivalence theorem reported in [Grosof, 1991], detailed in [Grosof, 1992b]) to circumscription with nonlayered prioritization.

Related Work: Note that we emphasize updating with new defaults, not just new for-sure axioms, unlike the conditional approaches to NMR (e.g., [Kraus et al., 1990]). The ideas and results here apply to other NM formalisms, e.g.: Default Logic and Poole's [1988] and Brewka's [1989] systems, via the equivalence result in [Lifschitz, 1990]; as well as Geffner's [1989] system. The closest idea to conjunctive decomposition in the previous NMR literature is [Rathmann, 1990], who focussed, however, on conjunctively integrating heterogeneously-specified circumscriptive theories. He considered, moreover, only layered-priority predicate circumscriptions. Rathmann's and our work was developed independently. We are unaware of any other applications of reformulation to non-monotonic reasoning.

More Decompositions and Safeties: We did not have space here to report a number of additional results [Grosof, 1992b] about decompositions and their implications for safeties of updating, including about higher prioritization, hypotheticals, syntactic positivity, "serial" decompositions, weaker forms of irrelevance; and about the relationship of decompositions to specification and backward inference.

A lurithms and Automation of Our Results: In fi: $:$ work, we plan to automate recognition of decomi. 'ons and safeties of updating $\mathrm{cf}$. our theorems, and : ctual performance of the according reformulation: or the disjoint-predicates and asocial-monadic cases, we have polynomial-time algorithms to perform this: $O\left(n^{3}\right)$ time, where $n$ is the size of the CLD axiom set.

Exploiting Truth Maintenance: Such recognition establishes "monotonicity" (i.e., implication) relationships between theories and sub-theories (e.g., theory after update versus theory before update; or theory versus slice). We plan also to automate a generalized ATMS-style [de Kleer, 1986] high-level architectural book-keeping scheme to exploit such stored monotonicity relationships to support inference and belief revision in a prioritized database. [Grosof, 1992b] gives details.

More General Cases of Disjoint Describability: In future work, we aim to find cases of disjoint describability that are more general than asocialmonadic, but are still easily :ecognizable syntactically (in terms of the syntax of the global axiom set). E.g., in 
our main example, it would be nice to be able to particularize the Meetings slice to the individual Ed, in the same way that the asocial monadic result guarantees one can particularize the Legged-ness slice to the individual Joe. Right now, we can show this particularization about Ed is legitimate, but our proof method is by hand. We would like to be able to formalize and automate a class of decompositions for which this (Ed etc.) is an instance.

Conclusions I: See Strategy and Summary in section 1.

Conclusions II: Analyzing Computational Advantages of Reformulation: In future work, we also plan to analyze in detail the computational advantages and trade-offs involved in our decompositions and definitional reformulations. You may be wondering why we did not give any such computational complexity analysis in this paper. The main reason is that the picture is quite complicated for non-monotonic reasoning.

Even for query-answering in propositional default theories without priorities, current results show worstcase is exponential (NP-hard) [Selman and Kautz, 1989] [Kautz and Selman, 1989] [Selman and Levesque, 1989]. Thus: Divide-to-conquer, i.e., seeking locality, is clearly desirable.

But the basic complexity results for any kind of forward reasoning with priorities, for any kind of belief revision, and even for most kinds of backward (queryanswering) reasoning are not available for circumscription, or other NM formalisms. Known tractable cases are highly restricted. ([Selman and Kautz, 1989] [Kautz and Selman, 1989] give polynomial-time backward procedures for special cases, including restrictions of Horn, of propositional default reasoning in their model-preference logic and in Default Logic. Delgrande [1991] gives a polynomial-time backward procedure for a Horn propositional case of his conditional logic.)

However, we believe that as these results bernme available, we will be able to show that decomposition and reformulation are advantageous. Our aim has been to develop methods that will be broadly applicable, and to break off a piece of the overall hard problems of non-monotonic reasoning. In current work, we are addressing how to relate our results to currently known tractable and intractable cases.

One clear advantage is that for many cases with quantification, for which worst-case is undecidable ([Reiter, 1980] [Kolaitis and Papadimitriou, 1988]): We are able to reformulate some of the reasoning to become propositional, hence decidable. E.g., when reasoning about individuals, for the asocially monadic class of theories (see Theorem 16).

\section{Acknowledgements}

Thanks to Devika Subramanian, Vladimir Lifschitz, and Michael Lowry for long-ago useful discussions on logical aspects of definitional reformulation. Thanks to Leora Morgenstern, Hector Geffner, and two anonymous reviewers for their comments on previous drafts.

\section{References}

A. Baker and M. Ginsberg. A theorem prover for prioritized circumscription. Proceedings $I J C A I-89$, pages 463-467, Detroit, MI., 1989.

G. Brewka. Preferred subtheories: An extended logical framework for default reasoning. Proceedings IJCAI-89, pages 1043-1049, Detroit, Michigan, 1989.

K. Clark. Negation as failure. In H. Gallaire and J. Minker, editors, Logic and Data Bases, pages 293322. Plenum Press, New York, 1978.

J. de Kleer. An assumption-based truth maintenance system. Artificial Intelligence, 28:280-297, 1986.

James P. Delgrande. Incorporating nonmonotonic reasoning in horn clause theories. Proceedings of AAAI-91, pages 405-411, 445 Burgess Drive, Menlo Park, CA 94025, 1991. AAAI Press.

J. Doyle. A truth maintenance system. Artificial Intelligence, 12:231-272, 1979.

H. Geffner. Default Reasoning: Causal and Conditional Theories. PhD thesis, Computer Science Department, UCLA, Los Angeles, CA, 1989. Revised version published by MIT Press, 1992.

M. Ginsberg. A circumscriptive theorem prover. Artificial Intelligence, 39:209-230, 1989.

Benjamin N. Grosof. Generalizing prioritization. Proceedings of the Second International Conference on Principle of Knowledge Representation and Reasoning, pages 289-300, April 1991. Also available as IBM Research Report RC15605, IBM T.J. Watson Research Center, P.O. Box 704, Yorktown Heights, NY 10598.

Benjamin N. Grosof. Generalizing prioritization ii (working title). Working paper., 1992.

Benjamin N. Grosof. Updating and Structure in NonMonotonic Theories. PhD thesis, Computer Science Dept., Stanford University, Stanford, California $94305,1992$.

Katsumi Inoue and Nicolas Helft. On theorem provers for circumscription. Working paper. This is a revised version of a paper appearing under the same title in the Proceedings of the Canadian Conference on Computer Science and Artificial Intelligence '90, Ottawa, Canada, May 1990., Apr 1990.

Katsumi Inoue, Nicolas Helft, and David Poole. Query answering in circumscription. Proceedings of IJCAI-91, pages 426-431, San Mateo, California, 1991. Morgan Kaufmann.

H. Kautz and B. Selman. Hard problems for simple default logics. Proceedings of the First International Conference on Principle of Knowledge Representa- 
tion and Reasoning, pages 189-197, Toronto, Ontario, 1989 .

Phokion G. Kolaitis and Christos H. Papadimitriou. Some computational aspects of circumscription. Proceedings of $A A A I-88$, pages 465-469, San Mateo, California, 1988. Morgan Kaufmann. Held Minneapolis, MN.

S. Kraus, D. Lehmann, and M. Magidor. Preferential models and cumulative logics. Artificial Intelligence, 44:167-207, 1990.

Vladimir Lifschitz. Some results on circumscription. Proceedings of the First AAAI Non-Monotonic Reasoning Workshop, pages 151-164, Oct 1984. Held New Paltz, NY.

V. Lifschitz. Computing circumscription. Proceedings IJCAI-85, pages 121-127, Los Angeles, CA. 85.

Vladimir Lifschitz. On the declarative ser ics of logic programs with negation. In Matthe. Jinsberg, editor, Readings in Nonmonotonic : ining. Morgan Kaufmann, San Mateo, CA, 1987.

V. Lifschitz. Circumscriptive theories: a logic-based framework for knowledge representation. Journal of Philosophical Logic, 17:391-441, 1988.

Vladimir Lifschitz. On open defaults. Proceedings Symposium on Computational Logic, Brussels, Belgium, 1990.

J. McCarthy. Applications of circumscription to formalizing commonsense knowledge. Artificial Intelligence, 28:89-116, 1986.

R. Moore. Semantical considerations on nonmonotonic logics. Artificial Intelligence, 25:75-94, 1985.

D. Poole. A logical framework for default reasoning. Artificial Intelligence, 36:27-47, 1988.

Teodor Przymusinski. On the declarative semantics of deductive databases and logic programs. In J. Minker, editor, Foundations of Deductive Databases and Logic Programming. Morgan Kaufmann, San Mateo, CA., 1988.

T. Przymusinski. An algorithm for circumscription. Artificial Intelligence, 38:49-73, 1989.

Peter K. Rathmann. Nonmonotonic Semantics for Partitioned Knowledge Bases. PhD thesis, Computer Science Dept., Stanford University, Stanford, California 94305, Jun 1990.

R. Reiter. A logic for default reasoning. Artificial Intelligence, 12:81-132, 1980.

B. Selman and H. Kautz. The complexity of model preference default theories. In M. Reinfrank et al., editor, Proceedings of the Second International Workshop on Non-Monotonic Reasoning, pages 115-130, Berlin, Germany, 1989. Springer Lecture Notes on Computer Science.
B. Selman and H. Levesque. The tractability of pathbased inheritance. Proceedings IJCAI-89, pages 11401145, Detroit, MI., 1989.

D. Touretzky. The Mathematics of Inheritance Systems. Pitman, London, 1986. 